\title{
Esophagus and Esophagogastric Junction Cancer PT1 TNM Finding v7
}

National Cancer Institute

\section{Source}

National Cancer Institute. Esophagus and Esophagogastric Junction Cancer pT 1 TNM

Finding V7. NCI Thesaurus. Code C89728.

Esophagus and esophagogastric junction cancer with tumor invading lamina propria,

muscularis mucosae, or submucosa. (from AJCC 7th Ed.) 